\title{
Old and New Reductions of Dispersionless Toda Hierarchy ${ }^{\star}$
}

\author{
Kanehisa TAKASAKI \\ Graduate School of Human and Environmental Studies, Kyoto University, \\ Yoshida, Sakyo, Kyoto, 606-8501, Japan \\ E-mail: takasaki@math.h.kyoto-u.ac.jp \\ URL: http://www.math.h.kyoto-u.ac.jp/ takasaki/
}

Received June 06, 2012, in final form December 15, 2012; Published online December 19, 2012

http://dx.doi.org/10.3842/SIGMA.2012.102

\begin{abstract}
This paper is focused on geometric aspects of two particular types of finitevariable reductions in the dispersionless Toda hierarchy. The reductions are formulated in terms of "Landau-Ginzburg potentials" that play the role of reduced Lax functions. One of them is a generalization of Dubrovin and Zhang's trigonometric polynomial. The other is a transcendental function, the logarithm of which resembles the waterbag models of the dispersionless KP hierarchy. They both satisfy a radial version of the Löwner equations. Consistency of these Löwner equations yields a radial version of the Gibbons-Tsarev equations. These equations are used to formulate hodograph solutions of the reduced hierarchy. Geometric aspects of the Gibbons-Tsarev equations are explained in the language of classical differential geometry (Darboux equations, Egorov metrics and Combescure transformations). Flat coordinates of the underlying Egorov metrics are presented.
\end{abstract}

Key words: dispersionless Toda hierarchy; finite-variable reduction; waterbag model; Landau-Ginzburg potential; Löwner equations; Gibbons-Tsarev equations; hodograph solution; Darboux equations; Egorov metric; Combescure transformation; Frobenius manifold; flat coordinates

2010 Mathematics Subject Classification: 35Q99; 37K10; 53B50; 53D45

\section{Introduction}

The notion of finite-variable reductions in dispersionless integrable hierarchies [24] has rich geometric contents that range from the classical differential geometry of orthogonal curvilinear coordinates [10] to the modern theory of Frobenius manifolds [12]. Moreover, the Löwner equations [25], first introduced to solve the Bieberbach conjecture in the univalent function theory, also play a fundamental role in this issue. In this paper, we consider the dispersionless Toda hierarchy [31]. Although a general scheme of finite-variable reductions in this case is already established [6, 26, 33, 34], finding interesting examples is another issue. We here report two examples, one being a generalization of an "old" example, and the other being a "new" one. These examples turn out to fit well into the aforementioned geometric perspectives. We believe that these examples have their own interesting features, part of which will be presented later on.

As in the case of other dispersionless integrable systems [24], a finite-variable reduction of the dispersionless Toda hierarchy can be characterized by a globally defined reduced Lax function $\lambda(p)$. Borrowing the terminology of topological field theories [11], let us call this reduced Lax function a "Landau-Ginzburg potential" ${ }^{1}$. The two examples addressed in this paper have Landau-Ginzburg potentials of the following form:

\footnotetext{
*This paper is a contribution to the Special Issue "Geometrical Methods in Mathematical Physics". The full collection is available at http://www.emis.de/journals/SIGMA/GMMP2012.html

${ }^{1}$ This is somewhat problematical, because there is no guarantee that the reduced Lax function has an associated topological field theory or a Frobenius manifold.
} 


\section{Case I:}

$$
\lambda(p)=p^{-N} \prod_{i=1}^{M}\left(p-b_{i}\right)^{\kappa_{i}}, \quad \text { where } \quad \sum_{i=1}^{M} \kappa_{i}-N>0, \quad \kappa_{i} \neq 0, \quad N \neq 0 .
$$

\section{Case II:}

$$
\lambda(p)=\prod_{i=1}^{M}\left(p-b_{i}\right)^{\kappa_{i}} \exp \left(\sum_{k=1}^{N} c_{k} p^{-k}\right), \quad \text { where } \sum_{i=1}^{M} \kappa_{i}>0, \quad \kappa_{i} \neq 0, \quad N>0 .
$$

(1.1) is a generalization of the well known trigonometric Landau-Ginzburg potential studied by Dubrovin and Zhang [16]. Dubrovin and Zhang's Landau-Ginzburg potential corresponds to the case where $N>0$ and $\kappa_{1}=\cdots=\kappa_{M}=1$. This case contains, as particular examples, the dispersionless limit of the 1D Toda and bigraded Toda hierarchies [5]. By allowing $\kappa_{i}$ 's and $N$ to take negative values, we can include therein, for example, the dispersionless limit of the Ablowitz-Ladik hierarchy [1] and its possible generalizations as well. Thus this apparently "old" case itself deserves to be studied in detail.

(1.2) is presumably a new example that has never been studied in the literature. This LandauGinzburg potential is not a rational function of $p$. In this respect, (1.2) is conceptually similar to the so called "waterbag" models that, too, have irrational Landau-Ginzburg potentials. The waterbag models were first presented in the celebrated work of Gibbons and Tsarev [21, 22] on reductions of the Benney hierarchy, and have been further studied in the dispersionless KP hierarchy from various points of view $[2,7,8,20,28]$. In the most general formulation presented by Ferguson and Strachan [20], the Landau-Ginzburg potential is a sum of a polynomial and logarithmic terms of the following form

$$
\lambda(p)=p^{N}+\sum_{k=2}^{N} c_{k} p^{N-2}+\sum_{i=1}^{M} \kappa_{i} \log \left(p-b_{i}\right)
$$

Analogy with the waterbag models becomes more manifest in the logarithmic form

$$
\log \lambda(p)=\sum_{i=1}^{M} \kappa_{i} \log \left(p-b_{i}\right)+\sum_{k=1}^{N} c_{k} p^{-k}
$$

of (1.2), which takes almost the same form as (1.3) except that the (Laurent) polynomial part of the latter is a polynomial in $p^{-1} .^{2}$ In this sense, one may think of (1.2) as a variation of the waterbag models.

Let us mention that there are a few proposals of waterbag models for the dispersionless Toda hierarchy. The earliest one is Yu's model [37 $]^{3}$. Seemingly unaware of Yu's work, Chang proposed three types of waterbag models [9]. (1.1) is a slight generalization of Chang's first model (which amounts to the case where $N=-1$, and for which Chang considered a Frobenius structure). Chang's second and third models are actually a generalization of Yu's model. The logarithmic expression (1.4) of our second case resembles Chang's second and third models. We, however, feel that the exponentiated form (1.2) is more natural than the logarithmic expression.

\footnotetext{
${ }^{2}$ Another delicate difference is that the polynomial part of (1.3) is monic (namely, the coefficient of the highest degree term is 1) and has no next-highest degree term. In contrast, the (Laurent) polynomial part of (1.4) is not monic, and has no constant term.

${ }^{3}$ Actually, Yu considered a dispersionless limit of the discrete KP hierarchy. This hierarchy is a subsystem of the dispersionless Toda hierarchy, and one can readily translate Yu's waterbag model to the language of the latter.
} 
This paper is organized as follows. Section 2 is devoted to the Lax formalism. We show, by a direct method, that (1.1) and (1.2) give consistent reductions of the Lax equations of the dispersionless Toda hierarchy. The two-variable cases turn out to contain some well known (as well as new) dispersionless integrable hierarchies. Section 3 is focused on the Löwner equations and the associated Gibbons-Tsarev equations. The Löwner equations relevant to the dispersionless Toda hierarchy are a "radial" version of the "chordal" Löwner equations in the aforementioned work of Gibbons and Tsarev [21, 22]. We show that (1.1) and (1.2) satisfy the radial Löwner equations, and use these equations to formulate the generalized hodograph method [35, 36] for these reductions. Section 4 presents some geometric implications of the Gibbons-Tsarev equations in the language of Darboux equations, Egorov metrics and Combescure transformations. We show that three particular Egorov metrics underlie the radial Gibbons-Tsarev equations. Section 5 deals with flat coordinates of the Egorov metrics. Results in this section is somewhat restrictive. We encounter difficulties in generalizing the dual pair of Frobenius structures of Dubrovin and Zhang [16] to the more general Landau-Ginzburg potential (1.1). Moreover, we can construct only a single Frobenius structure without a dual for the Landau-Ginzburg potential (1.2). The method and the result for the latter, however, exhibits remarkable similarities with the work of Ferguson and Strachan [20].

\section{Lax equations}

\subsection{Lax formalism of dispersionless Toda hierarchy}

The Lax equations of the dispersionless Toda hierarchy [31] are formulated by two Lax functions $^{4} z(p), \bar{z}(p)$ of a spatial variable $s$, a momentum variable $p$, and two sets of time variables $\boldsymbol{t}=\left(t_{1}, t_{2}, \ldots\right)$ and $\overline{\boldsymbol{t}}=\left(\bar{t}_{1}, \bar{t}_{2}, \ldots\right){ }^{5} \quad p$ is a "classical limit" of the shift operator $e^{\partial / \partial s}$ that satisfies the twisted canonical commutation relation

$$
\left[e^{\partial / \partial s}, s\right]=e^{\partial / \partial s}
$$

In the classical (or "long-wave") limit, this commutation relation turns into the Poisson commutation relation

$$
\{p, s\}=p
$$

This Poisson bracket can be extended to arbitrary functions of $s$ and $p$ as

$$
\{f, g\}=p\left(\frac{\partial f}{\partial p} \frac{\partial g}{\partial s}-\frac{\partial f}{\partial s} \frac{\partial g}{\partial p}\right)
$$

In the most general formulation, the Lax functions $z(p)$ and $\bar{z}(p)$ are understood to be mutually independent formal Laurent series of $p$ of the form

$$
z(p)=p+u_{1}+u_{2} p^{-1}+\cdots, \quad \bar{z}(p)=\bar{u}_{0} p^{-1}+\bar{u}_{1}+\bar{u}_{2} p+\cdots .
$$

The coefficients $u_{n}=u_{n}(s, \boldsymbol{t}, \overline{\boldsymbol{t}})$ and $\bar{u}_{n}=\bar{u}_{n}(s, \boldsymbol{t}, \overline{\boldsymbol{t}})$ are dynamical variables. The leading coefficient $\bar{u}_{0}$ is assumed to take the exponential form

$$
\bar{u}_{0}=e^{\phi}, \quad \phi=\phi(s, \boldsymbol{t}, \overline{\boldsymbol{t}}) .
$$

\footnotetext{
${ }^{4} z$ and $\bar{z}$ amount to $\mathcal{L}$ and $\overline{\mathcal{L}}^{-1}$ in our previous notations [31].

${ }^{5}$ Throughout this paper, the overline "-" does not mean complex conjugation. For example, $t_{n}$ and $\bar{t}_{n}$ are independent variables.
} 
Let us define the polynomials $B_{n}(p)$ and $\bar{B}_{n}(p)$ in $p, p^{-1}$ as

$$
B_{n}(p)=\left(z(p)^{n}\right)_{\geq 0}, \quad \bar{B}_{n}(p)=\left(\bar{z}(p)^{n}\right)_{<0},
$$

where ()$_{\geq 0}$ and ()$_{<0}$ are projection operators acting on the linear space of Laurent series as

$$
\left(\sum_{n=-\infty}^{\infty} a_{n} p^{n}\right)_{\geq 0}=\sum_{n \geq 0} a_{n} p^{n}, \quad\left(\sum_{n=-\infty}^{\infty} a_{n} p^{n}\right)_{<0}=\sum_{n<0} a_{n} p^{n} .
$$

Time evolutions are generated by the Lax equations

$$
\begin{array}{lll}
\frac{\partial z(p)}{\partial t_{n}}=\left\{B_{n}(p), z(p)\right\}, & \frac{\partial z(p)}{\partial \bar{t}_{n}}=\left\{\bar{B}_{n}(p), z(p)\right\}, \\
\frac{\partial \bar{z}(p)}{\partial t_{n}}=\left\{B_{n}(p), \bar{z}(p)\right\}, & \frac{\partial \bar{z}(p)}{\partial \bar{t}_{n}}=\left\{\bar{B}_{n}(p), \bar{z}(p)\right\} .
\end{array}
$$

It is convenient to introduce the complementary generators

$$
B_{n}^{c}(p)=\left(z(p)^{n}\right)_{<0}, \quad \bar{B}_{n}^{c}(p)=\left(\bar{z}(p)^{n}\right)_{\geq 0}
$$

as well. The Lax equations can be thereby rewritten as

$$
\begin{array}{rlrl}
\frac{\partial z(p)}{\partial t_{n}}=\left\{z(p), B_{n}^{c}(p)\right\}, & & \frac{\partial z(p)}{\partial \bar{t}_{n}}=\left\{z(p), \bar{B}_{n}^{c}(p)\right\}, \\
\frac{\partial \bar{z}(p)}{\partial t_{n}}=\left\{\bar{z}(p), B_{n}^{c}(p)\right\}, & \frac{\partial \bar{z}(p)}{\partial \bar{t}_{n}}=\left\{\bar{z}(p), \bar{B}_{n}^{c}(p)\right\} .
\end{array}
$$

\subsection{Landau-Ginzburg potential as reduced Lax function}

We now specialize the Lax equations to the case where the formal (or local) Lax functions $z(p)$ and $\bar{z}(p)$ are linked with the globally defined Landau-Ginzburg potential $\lambda(p)$ as follows:

(I) For the Landau-Ginzburg potential (1.1),

$$
z(p)=\lambda(p)^{1 / \tilde{M}} \quad \text { as } p \rightarrow \infty, \quad \bar{z}(p)=\lambda(p)^{1 / N} \quad \text { as } p \rightarrow 0,
$$

where $\tilde{M}=\sum_{i=1}^{M} \kappa_{i}-N$. Recall that $\tilde{M}$ is assumed to be positive. $N$ can be both positive and negative.

(II) For the Landau-Ginzburg potential (1.2),

$$
z(p)=\lambda(p)^{1 / \tilde{M}} \quad \text { as } p \rightarrow \infty, \quad \bar{z}(p)=(\log \lambda(p))^{1 / N} \quad \text { as } p \rightarrow 0,
$$

where $\tilde{M}=\sum_{i=1}^{M} \kappa_{i}$. Recall that $\tilde{M}$ and $N$ is assumed to be positive.

The Lax equations (2.1) and its complementary form (2.2) thus reduce to the Lax equations

$$
\begin{aligned}
& \frac{\partial \lambda(p)}{\partial t_{n}}=\left\{B_{n}(p), \lambda(p)\right\}=\left\{\lambda(p), B_{n}^{c}(p)\right\}, \\
& \frac{\partial \lambda(p)}{\partial \bar{t}_{n}}=\left\{\bar{B}_{n}(p), \lambda(p)\right\}=\left\{\lambda(p), \bar{B}_{n}^{c}(p)\right\}
\end{aligned}
$$

for $\lambda(p)$. We can confirm that this is a consistent reduction procedure in the following sense. 
Theorem 1. The Lax equations (2.3) are equivalent to a system of first order evolutionary equations of the form

$$
\frac{\partial b_{i}}{\partial t_{n}}=F_{i n}, \quad \frac{\partial b_{i}}{\partial \bar{t}_{n}}=\bar{F}_{i n}
$$

for the Landau-Ginzburg potential (1.1) and

$$
\frac{\partial b_{i}}{\partial t_{n}}=F_{i n}, \quad \frac{\partial c_{k}}{\partial t_{n}}=G_{k n}, \quad \frac{\partial b_{i}}{\partial \bar{t}_{n}}=\bar{F}_{i n}, \quad \frac{\partial c_{k}}{\partial \bar{t}_{n}}=\bar{G}_{k n}
$$

for the Landau-Ginzburg potential (1.2), where $F_{i n}, \bar{F}_{\text {in }}$, etc. are functions of $b_{1}, \ldots, b_{M}, c_{1}$, $\ldots, c_{N}$ and their first order derivatives with respect to $s$.

Proof. Since the two cases can be treated in the same way, let us consider the Case II only. We can rewrite the Lax equations (2.3) in terms of $\log \lambda(p)$ as

$$
\begin{aligned}
& \frac{\partial \log \lambda(p)}{\partial t_{n}}=\left\{B_{n}(p), \log \lambda(p)\right\}=\left\{\log \lambda(p), B_{n}^{c}(p)\right\}, \\
& \frac{\partial \log \lambda(p)}{\partial \bar{t}_{n}}=\left\{\bar{B}_{n}(p), \log \lambda(p)\right\}=\left\{\log \lambda(p), \bar{B}_{n}^{c}(p)\right\} .
\end{aligned}
$$

The left hand side are linear combinations of $1 /\left(p-b_{i}\right)$ 's and $p^{-k}$ 's:

$$
\begin{gathered}
\frac{\partial \log \lambda(p)}{\partial t_{n}}=-\sum_{i=1}^{M} \frac{\partial b_{i}}{\partial t_{n}} \frac{\kappa_{i}}{p-b_{i}}+\sum_{k=1}^{N} \frac{\partial c_{k}}{\partial t_{n}} p^{-k}, \\
\frac{\partial \log \lambda(p)}{\partial \bar{t}_{n}}=-\sum_{i=1}^{M} \frac{\partial b_{i}}{\partial \bar{t}_{n}} \frac{\kappa_{i}}{p-b_{i}}+\sum_{k=1}^{N} \frac{\partial c_{k}}{\partial \bar{t}_{n}} p^{-k} .
\end{gathered}
$$

Since $B_{n}(p)$ and $\bar{B}_{n}(p)$ are polynomials in $p$ and $p^{-1}$, the right hand sides are rational functions of $p$. Actually, because of the two complementary expressions, they turn out to be linear combinations of $1 /\left(p-b_{i}\right)$ 's and $p^{-k}$ 's. For example, $\left\{B_{n}(p), \log \lambda(p)\right\}$ can be expanded as

$$
\begin{aligned}
\left\{B_{n}(p), \log \lambda(p)\right\}= & \sum_{i=1}^{M}\left\{B_{n}(p), \kappa_{i} \log \left(p-b_{i}\right)\right\}+\sum_{k=1}^{N}\left\{B_{n}(p), c_{k} p^{-k}\right\} \\
= & \sum_{i=1}^{M} p\left(-\frac{\partial B_{n}(p)}{\partial p} \frac{\partial b_{j}}{\partial s} \frac{\kappa_{i}}{p-b_{i}}-\frac{\partial B_{n}(p)}{\partial s} \frac{\kappa_{i}}{p-b_{i}}\right) \\
& +\sum_{k=1}^{N} p\left(\frac{\partial B_{n}(p)}{\partial p} \frac{\partial c_{k}}{\partial s} p^{-k}+\frac{\partial B_{n}(p)}{\partial s} k c_{k} p^{-k-1}\right),
\end{aligned}
$$

which has first order poles at $p=b_{i}$, an $N$-th order pole at $p=0$ and no poles in the finite part of the Riemann sphere. On the other hand, since $B_{n}^{c}(p)=O\left(p^{-1}\right)$ as $p \rightarrow \infty$, the complementary expression $\left\{\log \lambda(p), B_{n}^{c}(p)\right\}$ is also $O\left(p^{-1}\right)$. Consequently, the right hand side of the Lax equation is a rational function of the form

$$
\left\{B_{n}(p), \log \lambda(p)\right\}=\left\{\log \lambda(p), B_{n}^{c}(p)\right\}=\sum_{i=1}^{M} \frac{\kappa_{i} F_{i n}}{p-b_{i}}+\sum_{k=1}^{N} G_{k n} p^{-k},
$$

where $F_{i n}$ 's and $G_{i n}$ 's are functions of $b_{i}$ 's, $c_{k}$ 's and their first order derivatives with respect to $s$. Thus the Lax equations reduce to evolution equations as stated in the theorem. 


\subsection{Examples: two-variable reductions}

The simplest nontrivial cases are two-variable reductions. They contain the following old and new examples of integrable hierarchies.

(i) Landau-Ginzburg potential (1.1) with $N=1, M=2, \kappa_{1}=\kappa_{2}=1$ :

$$
\lambda(p)=p^{-1}\left(p-b_{1}\right)\left(p-b_{2}\right)=p+b+c p^{-1} .
$$

This is the reduced Lax function of the dispersionless 1D Toda hierarchy. It is well known that this hierarchy plays a fundamental role in a wide range of issues of mathematical physics. In a literal sense, this Laurent polynomial is used for the Landau-Ginzburg (or "mirror") description of the topological sigma model of $\mathbf{C P}^{1}[17,18]$. The associated Frobenius structure is also a significant example of Dubrovin's duality [13, 29].

(ii) Landau-Ginzburg potential (1.1) with $N=-1, M=2, \kappa_{1}=1, \kappa_{2}=-1$ :

$$
\lambda(p)=p \frac{p-b}{p-c} .
$$

This is the reduced Lax function of the dispersionless Ablowitz-Ladik hierarchy. This hierarchy and the dispersive version have found new applications in universality classes of nonlinear waves [15] and local Gromov-Witten invariants of the resolved conifold [3, 4].

(iii) Landau-Ginzburg potential (1.2) with $N=M=1, \kappa_{1}=1$ :

$$
\lambda(p)=(p-b) \exp \left(c p^{-1}\right) .
$$

This seems to give a new dispersionless integrable hierarchy.

As regards the third example, one can further impose the condition $b=0$ and obtain a hierarchy with the reduced Lax function

$$
\lambda(p)=p \exp \left(c p^{-1}\right) .
$$

This is an interesting case in itself, because the inverse function of $\lambda(p)$ is Lambert's $W$-function that plays a role in the theory of Hurwitz numbers (see our previous paper [30] and references cited therein). This "one-variable reduction" can be generalized to Landau-Ginzburg potentials of the form

$$
\lambda(p)=p^{M} \exp \left(\sum_{k=1}^{N} c_{k} p^{-k}\right),
$$

where $M$ is an arbitrary positive integer. They should be classified as "Case III", though we shall not pursue this case in this paper.

\section{Löwner equations}

\subsection{General scheme of finite-variable reductions}

According to the general scheme [6, 26, 33, 34], finite-variable reductions of the dispersionless Toda hierarchy are characterized by the equations

$$
\frac{\partial z(p)}{\partial \lambda_{n}}=\frac{\alpha_{n} p}{p-\gamma_{n}} \frac{\partial z(p)}{\partial p}, \quad \frac{\partial \bar{z}(p)}{\partial \lambda_{n}}=\frac{\alpha_{n} p}{p-\gamma_{n}} \frac{\partial \bar{z}(p)}{\partial p}
$$


(referred to as "Löwner equations" in the following) for the reduced Lax functions

$$
z(p)=z\left(p ; \lambda_{1}, \ldots, \lambda_{K}\right), \quad \bar{z}(p)=\bar{z}\left(p ; \lambda_{1}, \ldots, \lambda_{K}\right)
$$

These equations are a variant of the radial Löwner equations that were first introduced by Löwner [25].

The reduced Lax functions depend on the space-time variables through the reduced dynamical variables $\lambda_{n}=\lambda_{n}(s, \boldsymbol{t}, \overline{\boldsymbol{t}})$. The reduced dynamical variables, in turn, are required to satisfy the "hydrodynamic system"

$$
\frac{\partial \lambda_{n}}{\partial t_{k}}=V_{k n} \frac{\partial \lambda_{n}}{\partial s}, \quad \frac{\partial \lambda_{n}}{\partial \bar{t}_{k}}=\bar{V}_{k n} \frac{\partial \lambda_{n}}{\partial s} .
$$

The "characteristic speeds" $V_{k n}=V_{k n}\left(\lambda_{1}, \ldots, \lambda_{K}\right)$ and $\bar{V}_{k n}=\bar{V}_{k n}\left(\lambda_{1}, \ldots, \lambda_{K}\right)$ are defined as

$$
V_{k n}=\gamma_{n} B_{k}^{\prime}\left(\gamma_{n}\right), \quad \bar{V}_{k n}=\gamma_{n} \bar{B}_{k}^{\prime}\left(\gamma_{n}\right),
$$

where the prime denotes the derivative, i.e., $B_{k}^{\prime}(p)=\partial B_{k}(p) / \partial p$ and $\bar{B}_{k}^{\prime}(p)=\partial \bar{B}_{k}(p) / \partial p$. Since $B_{1}(p)=p+u_{1}$, the characteristic speeds for the $t_{1}$-flow coincides with $\gamma_{n}$ :

$$
V_{1 n}=\gamma_{n} .
$$

Thus, once the reduced Lax functions are given as a solution of the Löwner equations, the Lax equations are transformed to the hydrodynamic system (3.3) for the "Riemann invariants" $\lambda_{1}, \ldots, \lambda_{K}$. A precise statement of this fact reads as follows $[6,26,33,34]$ :

Theorem 2. If $z(p)$ and $\bar{z}(p)$ satisfy the Löwner equations (3.6) with respect to $\lambda_{n}$ 's, and $\lambda_{n}$ 's satisfy the hydrodynamic system (3.3) with respect to the space-time variables, then $z(p)$ and $\bar{z}(p)$ satisfy the Lax equations (2.1) with respect to the space-time variables.

$\alpha_{n}$ 's and $\gamma_{n}$ 's in (3.1) are functions of $\left(\lambda_{1}, \ldots, \lambda_{K}\right)$ to be determined in the reduction procedure. Note that they are not arbitrary functions. Consistency of (3.1) yield the differential equations

$$
\frac{\partial \gamma_{n}}{\partial \lambda_{m}}=\frac{\alpha_{m} \gamma_{n}}{\gamma_{m}-\gamma_{n}}, \quad \frac{\partial \alpha_{n}}{\partial \lambda_{m}}=\frac{\alpha_{m} \alpha_{n}\left(\gamma_{m}+\gamma_{n}\right)}{\left(\gamma_{m}-\gamma_{n}\right)^{2}}, \quad m \neq n,
$$

which are referred as "Gibbons-Tsarev equations" or, more precisely, "radial Gibbons-Tsarev equations". These equations are a radial version of the celebrated Gibbons-Tsarev equations $[21,22]$ (see the remarks below).

It is interesting that the product and quotient of $\alpha_{n}, \gamma_{n}$ satisfy the following equations

$$
\frac{\partial}{\partial \lambda_{m}}\left(\alpha_{n} \gamma_{n}\right)=\frac{2\left(\alpha_{m} \gamma_{m}\right)\left(\alpha_{n} \gamma_{n}\right)}{\left(\gamma_{m}-\gamma_{n}\right)^{2}}, \quad \frac{\partial}{\partial \lambda_{m}}\left(\frac{\alpha_{n}}{\gamma_{n}}\right)=\frac{2 \gamma_{m} \gamma_{n}}{\left(\gamma_{m}-\gamma_{n}\right)^{2}} \frac{\alpha_{m}}{\gamma_{m}} \frac{\alpha_{n}}{\gamma_{n}} .
$$

The right hand side of these equations are symmetric with respect to $m$ and $n$. This implies the existence of potentials.

It is easy to identify these potentials. Expands both hand sides of (3.1) into Laurent series at $p=\infty$ and pick out the $p^{1}$ and $p^{2}$ terms from the first equation and the $p^{0}$ terms from the second equation. One can thus find the relations

$$
\alpha_{n}=\frac{\partial u_{1}}{\partial \lambda_{n}}, \quad \alpha_{n} \gamma_{n}=\frac{\partial u_{2}}{\partial \lambda_{n}}, \quad \frac{\alpha_{n}}{\gamma_{n}}=\frac{\partial \phi}{\partial \lambda_{n}},
$$


which show that $u_{1}, u_{2}$ and $\phi=\log \bar{u}_{0}$ play the role of potentials. By the first expression of $\alpha_{n}$ in (3.5), one can rewrite (3.1) as

$$
\frac{\partial z(p)}{\partial \lambda_{n}}=\frac{p z^{\prime}(p)}{p-\gamma_{n}} \frac{\partial u_{1}}{\partial \lambda_{n}}, \quad \frac{\partial \bar{z}(p)}{\partial \lambda_{n}}=\frac{p \bar{z}^{\prime}(p)}{p-\gamma_{n}} \frac{\partial u_{1}}{\partial \lambda_{n}} .
$$

It is these equations that can be obtained directly from the Lax equations under the finitevariable ansatz (3.2).

The problem is now converted to solving (3.3). This problem can be treated by the generalized hodograph method [35, 36].

Remark 1. The original form of the Gibbons-Tsarev equations [21, 22] read

$$
\frac{\partial \gamma_{n}}{\partial \lambda_{m}}=\frac{\alpha_{n}}{\gamma_{m}-\gamma_{n}}, \quad \frac{\partial \alpha_{n}}{\partial \lambda_{m}}=\frac{2 \alpha_{m} \alpha_{n}}{\left(\gamma_{m}-\gamma_{n}\right)^{2}}
$$

They are integrability conditions of the chordal Löwner equations

$$
\frac{\partial z(p)}{\partial \lambda_{n}}=\frac{\alpha_{n}}{p-\gamma_{n}} \frac{\partial z(p)}{\partial p}
$$

for the Lax function

$$
z(p)=p+u_{2} p^{-1}+\cdots
$$

of the Benney hierarchy or, more generally, of the dispersionless KP hierarchy [27]. Note that the $u_{1}$-term is absent here, and $u_{2}$ plays the role of a potential for the coefficients $\alpha_{n}$

$$
\alpha_{n}=\frac{\partial u_{2}}{\partial \lambda_{n}}
$$

Remark 2. Ferapontov et al. [19] presented the radial Gibbons-Tsarev equations (3.4) (written in a trigonometric form) in their work on finite-variable reductions of the Boyer-Finley equation. The Boyer-Finley equation is the lowest 2D part of the dispersionless Toda hierarchy.

\subsection{Hodograph solutions}

The generalized hodograph method $[35,36]$ is based on the fact that the characteristic speeds satisfy the equations

$$
\frac{1}{V_{k m}-V_{k n}} \frac{\partial V_{k n}}{\partial \lambda_{m}}=\frac{1}{\bar{V}_{k m}-\bar{V}_{k n}} \frac{\partial \bar{V}_{k n}}{\partial \lambda_{m}}=\frac{\alpha_{m} \gamma_{n}}{\left(\gamma_{m}-\gamma_{n}\right)^{2}}
$$

for $k=1,2, \ldots$. Note that these equations include the special case

$$
\frac{1}{\gamma_{m}-\gamma_{n}} \frac{\partial \gamma_{n}}{\partial \lambda_{m}}=\frac{\alpha_{m} \gamma_{n}}{\left(\gamma_{m}-\gamma_{n}\right)^{2}}
$$

associated with $V_{1 n}=\gamma_{n}$. One can derive these equations directly from the definition of $B_{k}(p)$ and $\bar{B}_{k}(p)$ [26] (see the remarks below) or by generating functions of these polynomials [6, 33]. Having these equations, one can readily apply the generalized hodograph method to the hydrodynamic system (3.3) as follows [26, 33, 34]: 
Theorem 3. If a set of functions $F_{n}=F_{n}\left(\lambda_{1}, \ldots, \lambda_{K}\right)$ satisfy the equations

$$
\frac{1}{F_{m}-F_{n}} \frac{\partial F_{n}}{\partial \lambda_{m}}=\frac{\alpha_{m} \gamma_{n}}{\left(\gamma_{m}-\gamma_{n}\right)^{2}}
$$

and the non-degeneracy condition

$$
\operatorname{det}\left(\frac{\partial F_{n}}{\partial \lambda_{m}}\right)_{m, n=1, \ldots, K} \neq 0
$$

then a solution of the hydrodynamic system (3.3) can be obtained from the hodograph relations

$$
s+\sum_{k \geq 1} t_{k} V_{k n}+\sum_{k \geq 1} \bar{t}_{k} \bar{V}_{k n}=F_{n}, \quad n=1, \ldots, K,
$$

as a $K$-tuple of implicit functions $\lambda_{n}=\lambda_{n}(s, \boldsymbol{t}, \overline{\boldsymbol{t}}), n=1, \ldots, K$, in a neighborhood of $(\boldsymbol{t}, \overline{\boldsymbol{t}})=$ $(\mathbf{0}, \mathbf{0})$.

Remark 3. The characteristic speeds $V_{k n}$ and $\bar{V}_{k n}$ have the contour integral representation

$$
V_{k n}=-\gamma_{n} \oint \frac{z(p)^{n}}{\left(p-\gamma_{n}\right)^{2}} \frac{d p}{2 \pi i}, \quad \bar{V}_{k n}=-\gamma_{n} \oint \frac{\bar{z}(p)^{n}}{\left(p-\gamma_{n}\right)^{2}} \frac{d p}{2 \pi i},
$$

where the contours encircle $p=\infty$ and $p=0$, respectively, leaving $\gamma_{n}$ outside. One can use the Löwner equations and the Gibbons-Tsarev equations to differentiate these contour integrals. Thus, after some algebra, one can derive (3.8).

Remark 4. Solutions of (3.10), too, can be obtained as contour integrals. For example, arbitrary linear combinations of $V_{k n}$ and $\bar{V}_{k n}$, which are obvious solutions of (3.10), can be cast into a contour integral of the form

$$
F_{n}=\gamma_{n} \oint \frac{F_{1}(z(p))}{\left(p-\gamma_{n}\right)^{2}} \frac{d p}{2 \pi i}+\gamma_{n} \oint \frac{F_{2}(\bar{z}(p))}{\left(p-\gamma_{n}\right)^{2}} \frac{d p}{2 \pi i},
$$

where $F_{1}(p)$ and $F_{2}(p)$ are arbitrary (analytic) functions. If $z(p)$ and $\bar{z}(p)$ are obtained from a globally defined Landau-Ginzburg potential $\lambda(p)$, one can unify the two integrals to a single integral

$$
F_{n}=\gamma_{n} \oint_{C} \frac{F(\lambda(p))}{\left(p-\gamma_{n}\right)^{2}} \frac{d p}{2 \pi i}
$$

along a general cycle $C$ in the domain of definition of $F(\lambda(p))$. This gives a more general solution of (3.10) as Ferapontov et al. [19] pointed out in their formulation.

\subsection{Löwner equations for Landau-Ginzburg potentials}

If the Lax functions $z(p)$ and $\bar{z}(p)$ are reduced to a single Landau-Ginzburg potential $\lambda(p)$, the Löwner equations (3.1) for the Lax functions, too, are reduced to the equations

$$
\frac{\partial \lambda(p)}{\partial \lambda_{n}}=\frac{\alpha_{n} p}{p-\gamma_{n}} \frac{\partial \lambda(p)}{\partial p}
$$

for $\lambda(p)$.

We show below that the Landau-Ginzburg potentials (1.1) and (1.2) do satisfy these equations. The relevant variables $\lambda_{n}$ are the critical values of $\lambda(p)$, i.e., the values of $\lambda(p)$ at the critical points $\gamma_{n}$ 's,

$$
\lambda_{n}=\lambda\left(\gamma_{n}\right), \quad \lambda^{\prime}\left(\gamma_{n}\right)=0, \quad n=1, \ldots, K,
$$


where $K=M$ in the case of (1.1) and $K=M+N$ in the case of (1.2). We choose these $\lambda_{n}$ 's as new coordinates on the parameter space of $\lambda(p)$, and treat $\lambda(p)$ as a function $\lambda\left(p ; \lambda_{1}, \ldots, \lambda_{K}\right)$ of $p$ and $\lambda_{n}$ 's.

Let us show a few technical remarks.

\section{Lemma 1.}

$$
\left.\frac{\partial \lambda(p)}{\partial \lambda_{m}}\right|_{p=\gamma_{n}}=\delta_{m n}
$$

Proof. By the definition (3.12) of $\lambda_{n}$ 's and the chain rule of differentiation,

$$
\delta_{m n}=\frac{\partial \lambda_{n}}{\partial \lambda_{m}}=\left.\frac{\partial \lambda(p)}{\partial \lambda_{m}}\right|_{p=\gamma_{n}}+\lambda^{\prime}\left(\gamma_{m}\right)=\left.\frac{\partial \lambda(p)}{\partial \lambda_{m}}\right|_{p=\gamma_{n}} .
$$

Lemma 2. If the Löwner equations (3.11) are satisfied, the coefficients $\alpha_{n}$ are uniquely determined by the equations themselves as

$$
\alpha_{n}=\frac{1}{\gamma_{n} \lambda^{\prime \prime}\left(\gamma_{n}\right)} \text {. }
$$

Proof. Let $p \rightarrow \gamma_{n}$ in (3.11). By (3.13), the left hand side tends to 1. As regards the right hand side,

$$
\lim _{p \rightarrow \gamma_{n}} \frac{\alpha_{n} p}{p-\gamma_{n}} \frac{\partial \lambda(p)}{\partial p}=\alpha_{n} \gamma_{n} \lim _{p \rightarrow \gamma_{n}} \frac{\lambda^{\prime}(p)}{p-\gamma_{n}}=\alpha_{n} \gamma_{n} \lambda^{\prime \prime}\left(\gamma_{n}\right) .
$$

Bearing these technical remarks in mind, let us examine the two cases separately.

Case I. It is convenient to consider the logarithmic derivative, rather than the derivative, of the Landau-Ginzburg potential (1.1):

$$
\frac{\partial \log \lambda(p)}{\partial p}=-\frac{N}{p}+\sum_{i=1}^{M} \frac{\kappa_{i}}{p-b_{i}}=\frac{Q(p)}{p \prod_{i=1}^{M}\left(p-b_{i}\right)} .
$$

The numerator $Q(p)$ is a polynomial of the form

$$
Q(p)=\tilde{M} p^{M}+\cdots \text {. }
$$

We assume that $Q(p)$ has $M$ distinct zeroes $\gamma_{n}, n=1, \ldots, M$,

$$
Q(p)=\tilde{M} \prod_{n=1}^{M}\left(p-\gamma_{n}\right), \quad \gamma_{m} \neq \gamma_{n} \quad \text { for } \quad m \neq n
$$

From now on, $\lambda(p)$ is understood to be a function of $p$ and $\lambda_{n}$ 's. The parameters $b_{i}$ 's, too, become functions of $\lambda_{n}$ 's. The derivative of $\log \lambda(p)$ with respect to $\lambda_{m}$ can be expressed as

$$
\frac{\partial \log \lambda(p)}{\partial \lambda_{m}}=-\sum_{i=1}^{M} \frac{\kappa_{i}}{p-b_{i}} \frac{\partial b_{i}}{\partial \lambda_{m}}=\frac{Q_{m}(p)}{\prod_{i=1}^{M}\left(p-b_{i}\right)},
$$

where $Q_{m}(p)$ is a polynomial of degree less than $M$. (3.15) and (3.16) imply the equality

$$
\frac{\partial \log \lambda(p)}{\partial \lambda_{m}}=\frac{Q_{m}(p) p}{Q(p)} \frac{\partial \log \lambda(p)}{\partial p}
$$


The problem is to find an explicit form of the pre-factor $Q_{m}(p) p / Q(p)$.

Since (3.13) implies that

$$
\left.\frac{\partial \log \lambda(p)}{\partial \lambda_{m}}\right|_{p=\gamma_{n}}=\left.\frac{1}{\lambda(p)} \frac{\partial \lambda(p)}{\partial \lambda_{m}}\right|_{p=\gamma_{n}}=\frac{\delta_{m n}}{\lambda_{n}}
$$

letting $p \rightarrow \gamma_{n}$ in (3.16) yields that

$$
Q_{m}\left(\gamma_{n}\right)=0 \quad \text { for } \quad n \neq m \text {. }
$$

Therefore, by the Lagrange interpolation formula, $Q_{m}(p) / Q(p)$ can be expressed as

$$
\frac{Q_{m}(p)}{Q(p)}=\sum_{n=1}^{M} \frac{Q_{m}\left(\gamma_{n}\right)}{Q^{\prime}\left(\gamma_{n}\right)\left(p-\gamma_{n}\right)}=\frac{Q_{m}\left(\gamma_{m}\right)}{Q^{\prime}\left(\gamma_{n}\right)\left(p-\gamma_{m}\right)} .
$$

Thus, defining $\alpha_{m}$ as

$$
\alpha_{m}=Q_{m}\left(\gamma_{m}\right) / Q^{\prime}\left(\gamma_{m}\right)
$$

we obtain the Löwner equations

$$
\frac{\partial \log \lambda(p)}{\partial \lambda_{m}}=\frac{\alpha_{m} p}{p-\gamma_{m}} \frac{\partial \log \lambda(p)}{\partial p}
$$

for $\log \lambda(p)$. Of course, they are equivalent to the Löwner equations (3.11) for $\lambda(p)$. By the second lemma above, $\alpha_{m}$ turns out to have another expression (3.14).

Case II. The logarithmic derivatives of the Landau-Ginzburg potential (1.2) can be expresses as

$$
\frac{\partial \log \lambda(p)}{\partial p}=\sum_{i=1}^{M} \frac{\kappa_{i}}{p-b_{i}}-\sum_{k=1}^{N} k c_{k} p^{-k-1}=\frac{Q(p)}{p^{N+1} \prod_{i=1}^{M}\left(p-b_{i}\right)}
$$

and

$$
\frac{\partial \log \lambda(p)}{\partial \lambda_{m}}=-\sum_{i=1}^{M} \frac{\kappa_{i}}{p-b_{i}} \frac{\partial b_{i}}{\partial \lambda_{m}}+\sum_{k=1}^{N} \frac{\partial c_{k}}{\partial \lambda_{m}} p^{-k}=\frac{Q_{m}(p)}{p^{N} \prod_{i=1}^{M}\left(p-b_{i}\right)},
$$

where $Q(p)$ is a polynomial of the form

$$
Q(p)=\tilde{M} \prod_{n=1}^{M+N}\left(p-\gamma_{n}\right), \quad \gamma_{m} \neq \gamma_{n} \quad \text { for } \quad m \neq n .
$$

$Q_{m}(p)$ is a polynomial of degree less than $M+N$, and the roots $\gamma_{n}$ of $Q(p)$ are assumed to be distinct. Starting from these data, one can derive the Löwner equations (3.11) in much the same way as in the Case I.

We thus obtain the following result:

Theorem 4. $\lambda(p)=\lambda\left(p ; \lambda_{1}, \ldots, \lambda_{K}\right)$ satisfies the Löwner equations (3.11) with the coefficients $\alpha_{n}$ defined by (3.14).

On the basis of this result, we can apply the foregoing scheme of finite-variable reductions to the Landau-Ginzburg potentials (1.1) and (1.2). 


\section{Darboux equations}

\subsection{Basic notions in classical differential geometry}

Given a diagonal metric $d s^{2}=\sum_{n=1}^{K}\left(h_{n} d \lambda_{n}\right)^{2}, 6$ one can define the rotation coefficients $\beta_{m n}$, $m \neq n$, as

$$
\beta_{m n}=\frac{1}{h_{m}} \frac{\partial h_{n}}{\partial \lambda_{m}}
$$

$h_{n}$ 's are called "Lamé coefficients" in the theory of orthogonal curvilinear coordinate systems $[10,35]$. The Riemann curvature of this metric vanishes if and only if the following equations are satisfied

$$
\begin{aligned}
& \frac{\partial \beta_{m n}}{\partial \lambda_{k}}=\beta_{m k} \beta_{k n} \quad \text { for } \quad k \neq m, n, \\
& \frac{\partial \beta_{m n}}{\partial \lambda_{m}}+\frac{\partial \beta_{m n}}{\partial \lambda_{n}}+\sum_{k=1}^{K} \beta_{k m} \beta_{k n}=0 .
\end{aligned}
$$

The first part (4.1) of these equations are called "Darboux equations" in the literature. Thus the Darboux equations are partial-flatness conditions, and have to be supplemented by the second equations (4.2) to ensure flatness.

If the rotation coefficients are symmetric, i.e., $\beta_{m n}=\beta_{n m}$, the metric components satisfy the conditions (Egorov conditions)

$$
\frac{\partial}{\partial \lambda_{m}}\left(h_{n}^{2}\right)=\frac{\partial}{\partial \lambda_{n}}\left(h_{m}^{2}\right)
$$

that ensure the existence of a potential $\phi$ (Egorov potential) such that

$$
h_{n}^{2}=\frac{\partial \phi}{\partial \lambda_{n}} .
$$

If the Darboux equations and the Egorov condition are satisfied, the flatness condition (4.2) reduces to the equations

$$
\sum_{k=1}^{K} \frac{\partial \beta_{m n}}{\partial \lambda_{k}}=0 .
$$

A diagonal metric that satisfies the Darboux equations and the Egorov conditions is called an "Egorov metric". Thus an Egorov metric is associated with a symmetric $\left(\beta_{m n}=\beta_{n m}\right)$ solution of the coupled system of the Darboux equations and (4.3). This system is closely related to the $K$-wave system $[12,14]$.

If one starts from a solution of the Darboux equations (4.1), the Lamé coefficients are recovered as a solution of the equations

$$
\frac{\partial h_{n}}{\partial \lambda_{m}}=h_{m} \beta_{m n} .
$$

The Darboux equations are integrability conditions of these linear equations. These equations leaves some arbitrariness in the Lamé coefficients. Two sets $h_{n}, \tilde{h}_{n}$ of Lamé coefficients have the same rotation coefficients if and only if their ratios $w_{n}=\tilde{h}_{n} / h_{n}$ satisfy the equations

$$
\frac{1}{w_{m}-w_{n}} \frac{\partial w_{n}}{\partial \lambda_{m}}=\frac{\partial \log h_{n}}{\partial \lambda_{m}}
$$

\footnotetext{
${ }^{6}$ We do not use Einstein's convention in this paper.
} 
Any solution of these equations thus gives a transformation on the set of Lamé coefficients with the same rotational coefficients. This transformation is called "Combescure transformation" in the theory of orthogonal curvilinear coordinate systems [10, 35].

\subsection{Implications of Gibbons-Tsarev equations}

Let us consider the Gibbons-Tsarev equations (3.4) in the language of Darboux equations and Egorov metrics. There are three metrics that are of particular interest:

$$
\begin{array}{ll}
\sum_{n=1}^{K}\left(h_{n} d \lambda_{n}\right)^{2}, & h_{n}=\sqrt{\alpha_{n} / \gamma_{n}}, \\
\sum_{n=1}^{K}\left(\tilde{h}_{n} d \lambda_{n}\right)^{2}, & \tilde{h}_{n}=\sqrt{\alpha_{n} \gamma_{n}}, \\
\sum_{n=1}^{K}\left(\hat{h}_{n} d \log \lambda_{n}\right)^{2}, \quad \hat{h}_{n}=\sqrt{\alpha_{n} \lambda_{n} / \gamma_{n}} .
\end{array}
$$

(4.5) and (4.6) underlie the hodograph solutions of the hydrodynamic equations (3.3). (4.5) and (4.7) are the metrics that we shall consider in the next section in the context of Frobenius structures.

We show below that the rotation coefficients of these metrics are symmetric and satisfy the Darboux equations (with respect to $\lambda_{n}$ 's in the first and second cases and $\log \lambda_{n}$ 's in the third case). Egorov potentials themselves can be readily identified as one can see from (3.5):

$$
h_{n}^{2}=\frac{\partial \phi}{\partial \lambda_{n}}, \quad \tilde{h}_{n}^{2}=\frac{\partial u_{2}}{\partial \lambda_{n}}, \quad \hat{h}_{n}^{2}=\frac{\partial \phi}{\partial \log \lambda_{n}} .
$$

The first two cases (4.5) and (4.6) are closely related.

Theorem 5. The Lamé coefficients of (4.5) and (4.6) have the same rotation coefficients

$$
\beta_{m n}=\beta_{n m}=\frac{\sqrt{\alpha_{m} \alpha_{n} \gamma_{m} \gamma_{n}}}{\left(\gamma_{m}-\gamma_{n}\right)^{2}} .
$$

These rotation coefficients satisfy the Darboux equations (4.1).

Proof. Do straightforward calculations using the Gibbons-Tsarev equations (3.4). The rotation coefficients of $h_{n}$ 's can be calculated as

$$
\begin{aligned}
\frac{1}{h_{m}} \frac{\partial h_{n}}{\partial \lambda_{m}} & =\frac{h_{n}}{h_{m}} \frac{\partial \log h_{n}}{\partial \lambda_{m}}=\sqrt{\frac{\alpha_{n} \gamma_{m}}{\alpha_{m} \gamma_{n}}}\left(\frac{1}{2 \alpha_{n}} \frac{\partial \alpha_{n}}{\partial \lambda_{m}}-\frac{1}{2 \gamma_{n}} \frac{\partial \gamma_{n}}{\partial \lambda_{m}}\right) \\
& =\sqrt{\frac{\alpha_{n} \gamma_{m}}{\alpha_{m} \gamma_{n}}}\left(\frac{1}{2 \alpha_{n}} \frac{\alpha_{m} \alpha_{n}\left(\gamma_{m}+\gamma_{n}\right)}{\left(\gamma_{m}-\gamma_{n}\right)^{2}}-\frac{1}{2 \gamma_{n}} \frac{\alpha_{m} \gamma_{n}}{\gamma_{m}-\gamma_{n}}\right)=\frac{\sqrt{\alpha_{m} \alpha_{n} \gamma_{m} \gamma_{n}}}{\left(\gamma_{m}-\gamma_{n}\right)^{2}} .
\end{aligned}
$$

In much the same way, the rotation coefficients of $\tilde{h}_{n}$ 's can be calculated as

$$
\begin{aligned}
\frac{1}{\tilde{h}_{n}} \frac{\partial \tilde{h}_{n}}{\partial \lambda_{m}} & =\frac{\tilde{h}_{n}}{\tilde{h}_{m}} \frac{\partial \log \tilde{h}_{n}}{\partial \lambda_{m}}=\sqrt{\frac{\alpha_{n} \gamma_{n}}{\alpha_{m} \gamma_{m}}}\left(\frac{1}{2 \alpha_{n}} \frac{\partial \alpha_{n}}{\partial \lambda_{m}}+\frac{1}{2 \gamma_{n}} \frac{\partial \gamma_{n}}{\partial \lambda_{m}}\right) \\
& =\sqrt{\frac{\alpha_{n} \gamma_{n}}{\alpha_{m} \gamma_{m}}}\left(\frac{1}{2 \alpha_{n}} \frac{\alpha_{m} \alpha_{n}\left(\gamma_{m}+\gamma_{n}\right)}{\left(\gamma_{m}-\gamma_{n}\right)^{2}}+\frac{1}{2 \gamma_{n}} \frac{\alpha_{m} \gamma_{n}}{\gamma_{m}-\gamma_{n}}\right)=\frac{\sqrt{\alpha_{m} \alpha_{n} \gamma_{m} \gamma_{n}}}{\left(\gamma_{m}-\gamma_{n}\right)^{2}} .
\end{aligned}
$$

Thus the rotation coefficients of $h_{n}$ 's and $\tilde{h}_{n}$ 's turn out to coincide. Differentiating them with respect to $\lambda_{k}$ and doing some algebra, one can derive the Darboux equations. 
Corollary 1. $\tilde{h}_{n}$ 's are a Combescure transformation of $h_{n}$ 's, and the ratios $\gamma_{n}=\tilde{h}_{n} / h_{n}$ satisfy (4.4).

The right hand side of (4.4) can be calculated explicitly as

$$
\frac{\partial \log h_{n}}{\partial \lambda_{m}}=\frac{\alpha_{m} \gamma_{n}}{\left(\gamma_{m}-\gamma_{n}\right)^{2}}
$$

thus (4.4) coincides with (3.9). Since (3.9) is a special case of (3.8), the characteristic speeds $V_{k n}$ and $\bar{V}_{k n}$, too, generate Combescure transformations of $h_{n}$. An analogous fact is known for the dispersionless KP hierarchy [27] and universal Whitham hierarchy [23] as well (see the remark below). Thus, as stressed by Tsarev [35], the notion of Combescure transformations lies in the heart of integrability of hydrodynamic systems such as (3.3).

The third case (4.7) is of somewhat different nature. It is $\log \lambda_{n}$ 's rather than $\lambda_{n}$ 's that are used to formulate the Darboux equations.

Theorem 6. The rotation coefficients

$$
\hat{\beta}_{m n}=\frac{1}{\hat{h}_{m}} \frac{\partial \hat{h}_{n}}{\partial \log \lambda_{m}}, \quad m \neq n,
$$

of the Lamé coefficients of (4.7) are related to (4.8) as

$$
\hat{\beta}_{m n}=\sqrt{\lambda_{m} \lambda_{n}} \beta_{m n},
$$

and satisfy the Darboux equations

$$
\frac{\partial \hat{\beta}_{m n}}{\partial \log \lambda_{k}}+\hat{\beta}_{m k} \hat{\beta}_{k n}=0 \quad \text { for } \quad k \neq m, n .
$$

Proof. Do straightforward calculations. Note, in particular, that the left hand side of (4.9) and (4.1) are related as

$$
\frac{\partial \hat{\beta}_{m n}}{\partial \log \lambda_{k}}+\hat{\beta}_{m k} \hat{\beta}_{k n}=\sqrt{\lambda_{m} \lambda_{n}} \lambda_{k}\left(\frac{\partial \beta_{m n}}{\partial \lambda_{k}}+\beta_{m k} \beta_{k n}\right) .
$$

Remark 5. If the rotation coefficients $\hat{\beta}_{m n}$ are homogeneous functions of $\lambda_{k}$ 's of degree zero, the Darboux equations (4.9) and the Euler equations

$$
\sum_{k=1}^{N} \frac{\partial \hat{\beta}_{m n}}{\partial \log \lambda_{k}}=\sum_{k=1}^{N} \lambda_{k} \frac{\partial \hat{\beta}_{m n}}{\partial \lambda_{k}}=0
$$

imply flatness of (4.7). This is indeed the case for the metrics $($,$) considered in the next$ section.

Remark 6. In the chordal case (3.7), the two sets $h_{n}=\sqrt{\alpha_{n}}, \tilde{h}_{n}=\sqrt{\alpha_{n}} \gamma_{n}$ of Lamé coefficients amount to (4.5) and (4.6). They have the same rotation coefficients

$$
\beta_{m n}=\beta_{n m}=\frac{\sqrt{\alpha_{m} \alpha_{n}}}{\left(\gamma_{m}-\gamma_{n}\right)^{2}}
$$

that satisfy the Darboux equations (4.1). Consequently, $\tilde{h}_{n}$ 's are a Combescure transformation of $h_{n}$ 's, and the ratios $\tilde{h}_{n} / h_{n}=\gamma_{n}$ satisfy the equations

$$
\frac{1}{\gamma_{m}-\gamma_{n}} \frac{\partial \gamma_{n}}{\partial \lambda_{m}}=\frac{\partial \log h_{n}}{\partial \lambda_{m}}=\frac{\alpha_{m}}{\left(\gamma_{m}-\gamma_{n}\right)^{2}} .
$$

The last equations are fundamental equations in the hodograph solutions of the Benney equations [21, 22], the dispersionless KP hierarchy [27] and the universal Whitham hierarchy [23, 32]. 


\section{Flat coordinates}

\subsection{Flat coordinates in Case I}

Let us recall Dubrovin and Zhang's construction [16] of two Frobenius structures on the parameter space of the Landau-Ginzburg potential

$$
\lambda(p)=p^{-N} \prod_{i=1}^{N}\left(p-b_{i}\right) .
$$

The Frobenius structures are realized by the following inner products (or metrics) $\langle\rangle,,($,$) and$ cubic forms $\langle,\rangle,,(,$,$) for vector fields on the parameter space of the Laurent polynomial:$

$$
\begin{aligned}
& \left\langle\partial, \partial^{\prime}\right\rangle=\sum_{n=1}^{M} \operatorname{res}_{p=\gamma_{n}}\left[\frac{\partial \lambda(p) \cdot \partial^{\prime} \lambda(p)}{d \lambda(p)}(d \log p)^{2}\right], \\
& \left\langle\partial, \partial^{\prime}, \partial^{\prime \prime}\right\rangle=\sum_{n=1}^{M} \operatorname{res}_{p=\gamma_{n}}\left[\frac{\partial \lambda(p) \cdot \partial^{\prime} \lambda(p) \cdot \partial^{\prime \prime} \lambda(p)}{d \lambda(p)}(d \log p)^{2}\right], \\
& \left(\partial, \partial^{\prime}\right)=\sum_{n=1}^{M} \operatorname{res}_{p=\gamma_{n}}\left[\frac{\partial \log \lambda(p) \cdot \partial^{\prime} \log \lambda(p)}{d \log \lambda(p)}(d \log p)^{2}\right], \\
& \left(\partial, \partial^{\prime}, \partial^{\prime \prime}\right)=\sum_{n=1}^{M} \operatorname{res}_{p=\gamma_{n}}\left[\frac{\partial \log \lambda(p) \cdot \partial^{\prime} \log \lambda(p) \cdot \partial^{\prime \prime} \log \lambda(p)}{d \log \lambda(p)}(d \log p)^{2}\right] .
\end{aligned}
$$

The cubic forms are used to define two commutative and associative product structures $\circ, \star$ of vector fields

$$
\left\langle\partial \circ \partial^{\prime}, \partial^{\prime \prime}\right\rangle=\left\langle\partial, \partial^{\prime} \circ \partial^{\prime \prime}\right\rangle=\left\langle\partial, \partial^{\prime}, \partial^{\prime \prime}\right\rangle, \quad\left(\partial \star \partial^{\prime}, \partial^{\prime \prime}\right)=\left(\partial, \partial^{\prime} \star \partial^{\prime \prime}\right)=\left(\partial, \partial^{\prime}, \partial^{\prime \prime}\right) .
$$

These two Frobenius structures are a prototype of Dubrovin's duality [13, 29].

When $\partial, \partial^{\prime}, \partial^{\prime \prime}$ are derivatives in $\lambda_{n}$ 's, one can use the Löwner equations (3.11) to evaluate these inner products and cubic forms as follows

$$
\begin{array}{rlrl}
\left\langle\frac{\partial}{\partial \lambda_{m}}, \frac{\partial}{\partial \lambda_{n}}\right\rangle & =\delta_{m n} \frac{\alpha_{n}}{\gamma_{n}}, & \left\langle\frac{\partial}{\partial \lambda_{k}}, \frac{\partial}{\partial \lambda_{m}}, \frac{\partial}{\partial \lambda_{n}}\right\rangle=\delta_{k m n} \frac{\alpha_{n}}{\gamma_{n}}, \\
\left(\frac{\partial}{\partial \lambda_{m}}, \frac{\partial}{\partial \lambda_{n}}\right)=\delta_{m n} \frac{\alpha_{n}}{\gamma_{n} \lambda_{n}}, & \left(\frac{\partial}{\partial \lambda_{k}}, \frac{\partial}{\partial \lambda_{m}}, \frac{\partial}{\partial \lambda_{n}}\right)=\delta_{k m n} \frac{\alpha_{n}}{\gamma_{n} \lambda_{n}^{2}},
\end{array}
$$

where $\delta_{k m n}=\delta_{k m} \delta_{m n}$ (i.e., $\delta_{k m n}$ is equal to 1 if $k=m=n$ and 0 otherwise). Thus (5.2) and (5.4) correspond to the Egorov metrics (4.5) and (4.7) considered in the last section. Note that (5.6) and (5.7) hold as far as the Löwner equations are satisfied. In particular, they are valid for the general case of (1.1) as well.

As shown by Dubrovin and Zhang [16], the first metric (5.2) has a system of flat coordinates $q_{1}, \ldots, q_{M-1}, \bar{q}_{0}, \bar{q}_{1}, \ldots, \bar{q}_{N}$ defined by the residue formula

$$
q_{n}=\underset{p=\infty}{\operatorname{res}}\left[\frac{z(p)^{n}}{n} d \log p\right], \quad \bar{q}_{0}=\phi, \quad \bar{q}_{n}=\underset{p=0}{\operatorname{res}}\left[\frac{\bar{z}(p)^{n}}{n} d \log p\right] .
$$

The inner products of $\partial / \partial q_{n}$ 's and $\partial / \partial \bar{q}_{n}$ 's are calculated explicitly as

$$
\left\langle\frac{\partial}{\partial q_{m}}, \frac{\partial}{\partial q_{n}}\right\rangle=\tilde{M} \delta_{m+n, \tilde{M}}, \quad\left\langle\frac{\partial}{\partial \bar{q}_{m}}, \frac{\partial}{\partial \bar{q}_{n}}\right\rangle=N \delta_{m+n, N}, \quad\left\langle\frac{\partial}{\partial q_{m}}, \frac{\partial}{\partial \bar{q}_{n}}\right\rangle=0 .
$$

Unfortunately, this construction of flat coordinates does not work for the more general Landau-Ginzburg potential (1.1). Actually, it seems likely that the metric (5.2) is no longer flat in other cases ${ }^{7}$. In this respect, it is very significant that Brini et al. [4] extended Dubrovin

\footnotetext{
${ }^{7}$ We thank one of the referees for pointing out this possibility.
} 
and Zhang's dual pair of Frobenius structures to the Lax function (2.4) of the dispersionless Ablowitz-Ladik hierarchy.

On the other hand, the construction of the second Frobenius structure is valid for the general case of (1.1) as well. This fact is shown by Chang [9] in the case where $N=-1$ and $\kappa_{i}$ 's are arbitrary. $\log b_{i}$ 's are flat coordinates of the second metric (5.4). One can confirm, with slightest modification of Chang's calculations, that this is also the case for an arbitrary value of $N$ as the following result of calculations of the inner product shows

$$
\left(\frac{\partial}{\partial b_{i}}, \frac{\partial}{\partial b_{j}}\right)=\left(1-\delta_{i j}\right) \frac{\kappa_{i} \kappa_{j}}{N b_{i} b_{j}}+\delta_{i j} \frac{\left(\kappa_{i}-N\right) \kappa_{i}}{N b_{i}^{2}}
$$

We omit details of these calculations, which are parallel to the proof of Lemma 3 below. Let us note that flatness of (5.8) is also a consequence of homogeneity of the rotation coefficients (cf. Remark 5).

Remark 7. Speaking more precisely, the definition of a Frobenius manifold requires some more data, in particular, an Euler vector field $E$ and associated scaling properties [12]. In the present setting, the Landau-Ginzburg potential $\lambda(p)$ has natural homogeneity, and one can use the vector field

$$
E=\sum_{n=1}^{M} \lambda_{n} \frac{\partial}{\partial \lambda_{n}}=\frac{1}{\tilde{M}} \sum_{i=1}^{M} b_{i} \frac{\partial}{\partial b_{i}}
$$

as an Euler vector field. Furthermore, one can introduce the prepotential $\mathcal{F}$ as a function of the flat coordinates $t_{n}$ and express the cubic form as

$$
\left(\frac{\partial}{\partial t_{l}}, \frac{\partial}{\partial t_{m}}, \frac{\partial}{\partial t_{n}}\right)=\frac{\partial^{3} \mathcal{F}}{\partial t_{l} \partial t_{m} \partial t_{n}}
$$

In the following, we refer to the notion of Frobenius manifolds in a loose sense, and focus our consideration on flatness of metrics.

\subsection{Flat coordinates in Case II}

We now turn to the Landau-Ginzburg potential (1.2). The goal is to present a set of flat coordinates for the inner product

$$
\left(\partial, \partial^{\prime}\right)=\sum_{n=1}^{M+N} \operatorname{res}_{p=\gamma_{n}}\left[\frac{\partial \log \lambda(p) \cdot \partial^{\prime} \log \lambda(p)}{d \log \lambda(p)}(d \log p)^{2}\right] .
$$

This inner product corresponds to the Egorov metric (4.7). Its flatness is ensured by homogeneity of the rotation coefficients (cf. Remarks 5 and 10). The associated cubic form is defined by

$$
\left(\partial, \partial^{\prime}, \partial^{\prime \prime}\right)=\sum_{n=1}^{M+N} \operatorname{res}_{p=\gamma_{n}}\left[\frac{\partial \log \lambda(p) \cdot \partial^{\prime} \log \lambda(p) \cdot \partial^{\prime \prime} \log \lambda(p)}{d \log \lambda(p)}(d \log p)^{2}\right],
$$

though we shall not study its implications. Let us mention that the technical details and the final result of the following consideration are remarkably similar to the case of Ferguson and Strachan [20].

Let us start from the natural coordinates $b_{1}, \ldots, b_{M}, c_{1}, \ldots, c_{N}$ of the parameter space. One can calculate part of the inner product explicitly as follows. 


\section{Lemma 3.}

$$
\left(\frac{\partial}{\partial b_{i}}, \frac{\partial}{\partial b_{j}}\right)=-\delta_{i j} \frac{\kappa_{i}}{b_{i}{ }^{2}}, \quad\left(\frac{\partial}{\partial b_{i}}, \frac{\partial}{\partial c_{k}}\right)=\delta_{k N} \frac{\kappa_{i}}{N b_{i} c_{N}}
$$

Proof. The derivative of $\log \lambda(p)$ with respect to $p$ is a function as shown in (3.17). The derivatives with respect to $b_{i}$ and $c_{k}$ take the simple form

$$
\frac{\partial \log \lambda(p)}{\partial b_{i}}=-\frac{\kappa_{i}}{p-b_{i}}, \quad \frac{\partial \log \lambda(p)}{\partial c_{k}}=p^{-k} .
$$

Consequently, the inner products in question can be expressed as

$$
\begin{aligned}
& \left(\frac{\partial}{\partial b_{i}}, \frac{\partial}{\partial b_{j}}\right)=\sum_{n=1}^{M+N} \operatorname{res}_{p=\gamma_{n}}\left[\frac{\kappa_{i} \kappa_{j}}{\left(p-b_{i}\right)\left(p-b_{j}\right)}\left(\frac{\partial \log \lambda(p)}{\partial p}\right)^{-1} \frac{d p}{p^{2}}\right], \\
& \left(\frac{\partial}{\partial b_{i}}, \frac{\partial}{\partial c_{k}}\right)=\sum_{n=1}^{M+N} \operatorname{res}_{p=\gamma_{n}}\left[-\frac{\kappa_{i} p^{-k}}{p-b_{i}}\left(\frac{\partial \log \lambda(p)}{\partial p}\right)^{-1} \frac{d p}{p^{2}}\right] .
\end{aligned}
$$

Since

$$
\left(\frac{\partial \log \lambda(p)}{\partial p}\right)^{-1}=\frac{p^{N+1} \prod_{k=1}^{M}\left(p-b_{k}\right)}{Q(p)}, \quad Q(p)=\tilde{M} \prod_{n=1}^{M+N}\left(p-\gamma_{n}\right),
$$

the 1-forms in the residues are rational and have poles of the first order at $p=\gamma_{1}, \ldots, \gamma_{M+N}$. Other possible poles are located at $p=b_{i}, b_{j}, 0$. The latter poles, however, can disappear because of zeros of the numerator in this expression of $(\partial \log \lambda(p) / \partial p)^{-1}$. For example, if $i \neq j$, the first 1 -form is non-singular at $p=b_{i}, b_{j}$ as well as at $p=0$. Since the residue theorem says that the sum of all residues is equal to 0 , one can conclude that

$$
\left(\frac{\partial}{\partial b_{i}}, \frac{\partial}{\partial b_{j}}\right)=0 \quad \text { for } \quad i \neq j \text {. }
$$

By the same reasoning, one can confirm that

$$
\left(\frac{\partial}{\partial b_{i}}, \frac{\partial}{\partial c_{k}}\right)=0 \quad \text { for } \quad k<N .
$$

As regards the remaining cases, one can use the residue theorem to rewrite the inner products as

$$
\begin{aligned}
& \left(\frac{\partial}{\partial b_{i}}, \frac{\partial}{\partial b_{i}}\right)=-\operatorname{res}_{p=b_{i}}\left[\frac{\kappa_{i}{ }^{2}}{\left(p-b_{i}\right)^{2}}\left(\frac{\partial \log \lambda(p)}{\partial p}\right)^{-1} \frac{d p}{p^{2}}\right], \\
& \left(\frac{\partial}{\partial b_{i}}, \frac{\partial}{\partial c_{N}}\right)=-\operatorname{res}_{p=0}\left[-\frac{\kappa_{i} p^{-N}}{p-b_{i}}\left(\frac{\partial \log \lambda(p)}{\partial p}\right)^{-1} \frac{d p}{p^{2}}\right] .
\end{aligned}
$$

In view of the local expression

$$
\left(\frac{\partial \log \lambda(p)}{\partial p}\right)^{-1}=\frac{p-b_{i}}{\kappa_{i}}+O\left(\left(p-b_{i}\right)^{2} \quad \text { as } \quad p \rightarrow b_{i}\right.
$$

and

$$
\left(\frac{\partial \log \lambda(p)}{\partial p}\right)^{-1}=-\frac{p^{N+1}}{N c_{N}}+O\left(p^{N+2}\right) \quad \text { as } \quad p \rightarrow 0,
$$

one can readily calculate the residues and confirm the statement for the remaining cases. 
This lemma shows that $\log b_{i}$ 's are part of flat coordinates. If one can further find a partial change of coordinates $\left(c_{1}, \ldots, c_{N}\right) \rightarrow\left(\bar{q}_{0}, \ldots, \bar{q}_{N-1}\right)$ that is independent of $b_{i}$ 's and flat in themselves, the new coordinate system $\log b_{1}, \ldots, \log b_{M}, \bar{q}_{0}, \ldots, \bar{q}_{N-1}$ are totally flat. We shall show that

$$
\bar{q}_{0}=\phi, \quad \bar{q}_{n}=\operatorname{res}_{p=0}\left[\frac{\bar{z}(p)^{n}}{n} d \log p\right], \quad n=1, \ldots, N-1,
$$

give such coordinates.

To this end, we need to know some properties of $\bar{q}_{n}$ 's (which are defined for $n \geq N$ as well by the same residue formula). Let $\bar{p}(\zeta)$ denote the inverse function of $\zeta=\bar{z}(p)$. It has a Laurent expansion of the form

$$
\bar{p}(\zeta)=e^{\phi} \zeta^{-1}\left(1+\bar{u}_{1} \zeta^{-1}+\cdots\right) .
$$

Therefore $\log \bar{p}(\zeta)$ is also well-defined as a series of the form

$$
\log \bar{p}(\zeta)=-\log \zeta+\phi+\bar{u}_{1} \zeta^{-1}+\cdots .
$$

Lemma 4. $\bar{q}_{n}$ 's coincide with the coefficients of the expansion of $\log \bar{p}(\zeta)$ :

$$
\log \bar{p}(\zeta)=-\log \zeta+\bar{q}_{0}+\bar{q}_{1} \zeta^{-1}+\cdots+\bar{q}_{n} \zeta^{-n}+\cdots .
$$

Proof. One can rewrite the definition (5.9) of $\bar{q}_{n}$ 's as

$$
\begin{aligned}
\bar{q}_{n} & =\underset{p=0}{\operatorname{res}}\left[\frac{\bar{z}(p)^{n}}{n} d \log p\right]=\underset{\zeta=\infty}{\operatorname{res}}\left[\frac{\zeta^{n}}{n} d \log \bar{p}(\zeta)\right] \\
& =-\underset{\zeta=\infty}{\operatorname{res}}\left[\log \bar{p}(\zeta) d\left(\frac{\zeta^{n}}{n}\right)\right]=-\underset{\zeta=\infty}{\operatorname{res}}\left[\log \bar{p}(\zeta) \zeta^{n-1} d \zeta\right] .
\end{aligned}
$$

This implies that $\log \bar{p}(\zeta)$ has a Laurent expansion as (5.10) shows.

Lemma 5. For $n=1, \ldots, N-1, \bar{q}_{n}$ is a polynomial of $c_{N-n}, \ldots, c_{N-1}$ and $e^{-\phi}$ of the form

$$
\bar{q}_{n}=\frac{1}{N} e^{(n-N) \phi} c_{N-n}+\text { higher orders in } c_{N-n+1}, \ldots, c_{N-1}
$$

and $q_{N}$ is a function of $b_{i}$ 's only

$$
\bar{q}_{N}=\frac{1}{N} \log \prod_{i=1}^{M}\left(-b_{i}\right)^{\kappa_{i}}
$$

Proof. Since $\bar{z}(p)=(\log \lambda(p))^{1 / N}$ and $c_{N}=e^{N \phi}$, one can calculate its $n$-th power as a Laurent series of the form

$$
\bar{z}(p)^{n}=\left(e^{N \phi} p^{-N}+c_{N-1} p^{1-N}+\cdots+c_{1} p^{-1}+\log \prod_{i=1}^{M}\left(-b_{i}\right)+O(p)\right)^{n / N} .
$$

Extracting the $p^{0}$ term yields (5.11) and (5.12).

(5.11) implies that the map $\left(c_{1}, \ldots, c_{N}\right) \mapsto\left(\bar{q}_{0}, \ldots, \bar{q}_{N-1}\right)$ is invertible. We now choose $b_{i}$ 's and $\bar{q}_{0}, \ldots, \bar{q}_{N-1}$ as a new coordinate system on the parameter space of $\lambda(p)$, and consider $\lambda(p)$ to be a function of $p$ and these coordinates. 
Lemma 6. The derivatives of $\log \lambda(p)$ with respect to $\bar{q}_{n}$ 's can be expressed as

$$
\frac{\partial \log \lambda(p)}{\partial \bar{q}_{n}}=\left.\left(-\zeta^{-n}+O\left(\zeta^{-N-1}\right)\right)\right|_{\zeta=\bar{z}(p)} \frac{d \log \lambda(p)}{d \log p}
$$

in a neighborhood of $p=0$.

Proof. We can use the so called "thermodynamic identity" [12]

$$
\frac{\partial \log \lambda(p)}{\partial \bar{q}_{n}} d \log p=-\left.\frac{\partial \log \bar{p}(\zeta)}{\partial \bar{q}_{n}}\right|_{\zeta=\bar{z}(p)} d \log \lambda(p)
$$

to rewrite the derivatives of $\log \lambda(p)$ with respect to $\bar{q}_{n}$ 's as

$$
\frac{\partial \log \lambda(p)}{\partial \bar{q}_{n}}=-\left.\frac{\partial \log \bar{p}(\zeta)}{\partial \bar{q}_{n}}\right|_{\zeta=\bar{z}(p)} \frac{d \log \lambda(p)}{d \log p} .
$$

Let us recall the expansion (5.10) of $\log \bar{p}(\zeta)$. In this expansion, the coefficients of $\zeta^{0}, \ldots, \zeta^{N-1}$ are $\bar{q}_{n}$ 's themselves and, as (5.12) shows, the next leading coefficient $q_{N}$ is a function of $b_{i}$ 's only. Consequently,

$$
\frac{\partial \log \bar{p}(\zeta)}{\partial \bar{q}_{n}}=\zeta^{-n}+O\left(\zeta^{-N-1}\right) .
$$

Remark 8. This is a place where the technical details slightly deviate from the case of Ferguson and Strachan [20]. As in their case, the flat coordinates under construction are a mixture of the two types of coordinates, $b_{i}$ 's and $\bar{q}_{n}$ 's, presented in the Case I. There is, however, a delicate difference in the derivation of the vital equalities (5.13). In their case, they could derive these equalities in a rather straightforward manner. In our case, we need a small piece of extra consideration on the special structure of $q_{N}$ as explained above.

Theorem 7. The inner product of the derivatives in $b_{i}$ 's and $\bar{q}_{n}$ 's can be expressed as

$$
\left(\frac{\partial}{\partial b_{i}}, \frac{\partial}{\partial b_{j}}\right)=-\delta_{i j} \frac{\kappa_{i}}{b_{i}{ }^{2}}, \quad\left(\frac{\partial}{\partial \bar{q}_{m}}, \frac{\partial}{\partial \bar{q}_{n}}\right)=N \delta_{m+n, N}, \quad\left(\frac{\partial}{\partial b_{i}}, \frac{\partial}{\partial \bar{q}_{n}}\right)=-\delta_{n 0} \frac{\kappa_{i}}{b_{i}} .
$$

In particular, $\log b_{i}$ 's and $\bar{q}_{n}$ 's are flat coordinates.

Proof. Since the expression

$$
\frac{\partial \log \lambda(p)}{\partial b_{i}}=-\frac{\kappa_{i}}{p-b_{i}}
$$

of derivatives with respect to $b_{i}$ 's persists to be true, the foregoing calculations of the inner products of $\partial / \partial b_{i}$ 's are also valid. To consider the inner products containing $\partial / \partial \bar{q}_{n}$ 's, we note the equality

$$
\frac{\partial \log \lambda(p)}{\partial \bar{q}_{n}}=\sum_{k=1}^{N} \frac{\partial c_{k}}{\partial \bar{q}_{n}} p^{-k}
$$

as well. Thus the 1-forms in the expression

$$
\left(\frac{\partial}{\partial \bar{q}_{m}}, \frac{\partial}{\partial \bar{q}_{n}}\right)=\sum_{n=1}^{M+N} \operatorname{res}_{p=\gamma_{n}}\left[\frac{\partial \log \lambda(p)}{\partial \bar{q}_{m}} \frac{\partial \log \lambda(p)}{\partial \bar{q}_{n}}\left(\frac{\partial \log \lambda(p)}{\partial p}\right)^{-1} \frac{d p}{p^{2}}\right],
$$




$$
\left(\frac{\partial}{\partial b_{i}}, \frac{\partial}{\partial \bar{q}_{n}}\right)=\sum_{n=1}^{M+N} \operatorname{res}_{p=\gamma_{n}}\left[\frac{\partial \log \lambda(p)}{\partial b_{i}} \frac{\partial \log \lambda(p)}{\partial \bar{q}_{n}}\left(\frac{\partial \log \lambda(p)}{\partial p}\right)^{-1} \frac{d p}{p^{2}}\right]
$$

of the inner products are rational, and can have extra poles at $p=0$ in addition to the first order poles at $p=q_{n}$ 's. By the residue theorem, the sum over the residues at $q_{n}$ 's can be converted to the residues at $p=0$ :

$$
\begin{aligned}
& \left(\frac{\partial}{\partial \bar{q}_{m}}, \frac{\partial}{\partial \bar{q}_{n}}\right)=-\operatorname{res}_{p=0}\left[\frac{\partial \log \lambda(p)}{\partial \bar{q}_{m}} \frac{\partial \log \lambda(p)}{\partial \bar{q}_{n}}\left(\frac{\partial \log \lambda(p)}{\partial p}\right)^{-1} \frac{d p}{p^{2}}\right], \\
& \left(\frac{\partial}{\partial b_{i}}, \frac{\partial}{\partial \bar{q}_{n}}\right)=-\operatorname{res}_{p=0}\left[-\frac{\kappa_{i}}{p-b_{i}} \frac{\partial \log \lambda(p)}{\partial \bar{q}_{n}}\left(\frac{\partial \log \lambda(p)}{\partial p}\right)^{-1} \frac{d p}{p^{2}}\right] .
\end{aligned}
$$

To evaluate the residues of $p=0$, let us recall (5.13). The residues in the last equalities can be thereby evaluated as

$$
\begin{aligned}
\underset{p=0}{\operatorname{res}}[ & \left.\frac{\partial \log \lambda(p)}{\partial \bar{q}_{m}} \frac{\partial \log \lambda(p)}{\partial \bar{q}_{n}}\left(\frac{\partial \log \lambda(p)}{\partial p}\right)^{-1} \frac{d p}{p^{2}}\right] \\
& =\underset{p=0}{\operatorname{res}}\left[\left.\left(-\zeta^{-m}+O\left(\zeta^{-N-1}\right)\right)\left(-\zeta^{-n}+O\left(\zeta^{-N-1}\right)\right)\right|_{\zeta=\bar{z}(p)} d \log \lambda(p)\right] \\
& =\operatorname{res}_{\zeta=\infty}^{\operatorname{res}}\left[\left(-\zeta^{-m}+O\left(\zeta^{-N-1}\right)\right)\left(-\zeta^{-n}+O\left(\zeta^{-N-1}\right)\right) N \zeta^{N-1} d \zeta\right]=-N \delta_{m+n, N}
\end{aligned}
$$

and

$$
\begin{aligned}
& \underset{p=0}{\operatorname{res}}[ {\left[\frac{\partial \log \lambda(p)}{\partial b_{i}} \frac{\partial \log \lambda(p)}{\partial \bar{q}_{n}}\left(\frac{\partial \log \lambda(p)}{\partial p}\right)^{-1} \frac{d p}{p^{2}}\right] } \\
& \quad=\underset{p=0}{\operatorname{res}}\left[\left.\left(-\frac{\kappa_{i}}{p-b_{i}}\right)\left(-\zeta^{-n}+O\left(\zeta^{-N-1}\right)\right)\right|_{\zeta=\bar{z}(p)} d \log p\right]=\delta_{n 0} \frac{\kappa_{i}}{b_{i}} .
\end{aligned}
$$

This completes the proof.

Remark 9. As in the case of Ferguson and Strachan [20], we have been unable to find a dual Frobenius structure. A naive idea will be to consider the same inner product (5.2) and the cubic form (5.3) as used for Dubrovin and Zhang's Landau-Ginzburg potential. This, however does not work because the 1 -forms in the definition of the inner product and the cubic form have essential singularities at $p=0$, and one cannot use the residue theorem.

Remark 10. Unlike the Landau-Ginzburg potential of Ferguson and Strachan [20], our LandauGinzburg potential (1.2) has natural quasi-homogeneity. We can use the vector field

$$
E=\sum_{n=1}^{M+N} \lambda_{n} \frac{\partial}{\partial \lambda_{n}}=\frac{1}{\tilde{M}} \sum_{i=1}^{M} b_{i} \frac{\partial}{\partial b_{i}}+\frac{1}{\tilde{M}} \sum_{k=1}^{N} k c_{k} \frac{\partial}{\partial c_{k}}
$$

as an Euler vector field.

\section{Conclusion}

We have examined the two Landau-Ginzburg potentials (1.1) and (1.2) and the associated reductions of the dispersionless Toda hierarchy. (1.1) is a generalization of Dubrovin and Zhang's Landau-Ginzburg potential [16], and contains distinct examples such as the dispersionless Ablowitz-Ladik hierarchy [4]. (1.2) is a transcendental function, and its logarithm resembles Ferguson and Strachan's generalized waterbag model [20] of the dispersionless KP hierarchy.

We have observed that these quite different Landau-Ginzburg potentials have very similar features: 
- Consistency of the reduction can by confirmed by a direct method as shown in the proof of Theorem 1.

- The radial Löwner equations can be derived in much the same way as shown in the proof of Theorem 2. Once these equations are obtained, the generalized hodograph method works automatically and in a unified way.

- They have natural homogeneity. This ensures flatness of the third Egorov metric (4.7), hence of the inner products (5.4) and (5.8). Even the construction of flat coordinates of these inner products are partially similar as shown in the proof of Theorem 7 .

Meanwhile, we have encountered a rather complicated situation in the problem of flatness of the first Egorov metric (4.5) (equivalently, the inner product (5.2)) in the cases other than Dubrovin and Zhang's Landau-Ginzburg potential (5.1). It is well known that non-flatness of the Egorov metric implies non-locality of an underlying Hamiltonian structure [6]. Presumably, this issue should be considered case-by-case. Of particular interest is the reduced Lax function (2.4) of the dispersionless Ablowitz-Ladik hierarchy, which is shown to have a dual pair of Frobenius structures [4]. Extending their result to other specializations of (1.1) is an important open problem.

Our second Landau-Ginzburg potential (1.2), too, raises many open problems. Questions posed by Ferguson and Strachan [20] to the waterbag models of the dispersionless KP hierarchy can be restated for our model. Moreover, the degenerate case (2.5) of this Landau-Ginzburg potential will have its own special properties and applications.

\section{Acknowledgements}

We thank the referees for many valuable comments. This work is partly supported by JSPS Grants-in-Aid for Scientific Research No. 21540218 and No. 22540186 from the Japan Society for the Promotion of Science.

\section{References}

[1] Ablowitz M.J., Ladik J.F., Nonlinear differential-difference equations, J. Math. Phys. 16 (1975), 598-603.

[2] Bogdanov L.V., Konopelchenko B.G., Symmetry constraints for dispersionless integrable equations and systems of hydrodynamic type, Phys. Lett. A 330 (2004), 448-459, nlin.SI/0312013.

[3] Brini A., The local Gromov-Witten theory of $\mathbb{C P}^{1}$ and integrable hierarchies, Comm. Math. Phys. 313 (2012), 571-605, arXiv:1002.0582.

[4] Brini A., Carlet G., Rossi P., Integrable hierarchies and the mirror model of local $\mathbb{C P}^{1}$, Phys. D 241 (2012), 2156-2167, arXiv:1105.4508.

[5] Carlet G., The extended bigraded Toda hierarchy, J. Phys. A: Math. Gen. 39 (2006), 9411-9435, math-ph/0604024.

[6] Carlet G., Lorenzoni P., Raimondo A., The reductions of the dispersionless 2D Toda hierarchy and their Hamiltonian structures, J. Phys. A: Math. Theor. 43 (2010), 045201, 13 pages, arXiv:0910.1210.

[7] Chang J.-H., On the waterbag model of dispersionless KP hierarchy, J. Phys. A: Math. Gen. 39 (2006), 11217-11230, nlin.SI/0603007.

[8] Chang J.-H., On the waterbag model of the dispersionless KP hierarchy. II, J. Phys. A: Math. Theor. 40 (2007), 12973-12985, nlin.SI/0702014.

[9] Chang J.-H., Remarks on the waterbag model of dispersionless Toda hierarchy, J. Nonlinear Math. Phys. 15 (2008), suppl. 3, 112-123, arXiv:0709.3859.

[10] Darboux G., Leçons sur les systèmes orthogonaux et les coordonnées curvilignes, Gauthier-Villars, Paris, 1910.

[11] Dijkgraaf R., Intersection theory, integrable hierarchies and topological field theory, in New Symmetry Principles in Quantum Field Theory (Cargèse, 1991), NATO Adv. Sci. Inst. Ser. B Phys., Vol. 295, Plenum, New York, 1992, 95-158, hep-th/9291993. 
[12] Dubrovin B.A., Geometry of 2D topological field theories, in Integrable Systems and Quantum Groups (Montecatini Terme, 1993), Lecture Notes in Math., Vol. 1620, Springer, Berlin, 1996, 120-348, hep-th/9407018.

[13] Dubrovin B.A., On almost duality for Frobenius manifolds, in Geometry, topology, and mathematical physics, Amer. Math. Soc. Transl. Ser. 2, Vol. 212, Amer. Math. Soc., Providence, RI, 2004, 75-132, math.DG/0307374.

[14] Dubrovin B.A., On the differential geometry of strongly integrable systems of hydrodynamics type, Funct. Anal. Appl. 24 (1990), 280-285.

[15] Dubrovin B.A., On universality of critical behaviour in Hamiltonian PDEs, in Geometry, Topology, and Mathematical Physics, Amer. Math. Soc. Transl. Ser. 2, Vol. 224, Amer. Math. Soc., Providence, RI, 2008, 59-109, arXiv:0804.3790.

[16] Dubrovin B.A., Zhang Y., Extended affine Weyl groups and Frobenius manifolds, Compositio Math. 111 (1998), 167-219, hep-th/9611200.

[17] Eguchi T., Hori K., Yang S.K., Topological $\sigma$ models and large- $N$ matrix integral, Internat. J. Modern Phys. A 10 (1995), 4203-4224, hep-th/9503017.

[18] Eguchi T., Yang S.K., The topological $\mathbb{C P}^{1}$ model and the large- $N$ matrix integral, Modern Phys. Lett. A 9 (1994), 2893-2902, hep-th/9407134.

[19] Ferapontov E.V., Korotkin D.A., Shramchenko V.A., Boyer-Finley equation and systems of hydrodynamic type, Classical Quantum Gravity 19 (2002), L205-L210, gr-qc/0401118.

[20] Ferguson J.T., Strachan I.A.B., Logarithmic deformations of the rational superpotential/Landau-Ginzburg construction of solutions of the WDVV equations, Comm. Math. Phys. 280 (2008), 1-25, math-ph/0605078.

[21] Gibbons J., Tsarev S.P., Conformal maps and reductions of the Benney equations, Phys. Lett. A 258 (1999), 263-271.

[22] Gibbons J., Tsarev S.P., Reductions of the Benney equations, Phys. Lett. A 211 (1996), 19-24.

[23] Guil F., Mañas M., Martínez Alonso L., The Whitham hierarchies: reductions and hodograph solutions, J. Phys. A: Math. Gen. 36 (2003), 4047-4062, nlin.SI/0209051.

[24] Krichever I.M., The $\tau$-function of the universal Whitham hierarchy, matrix models and topological field theories, Comm. Pure Appl. Math. 47 (1994), 437-475, hep-th/9205110.

[25] Löwner K., Untersuchungen über schlichte konforme Abbildungen des Einheitskreises. I, Math. Ann. 89 (1923), 103-121.

[26] Mañas M., S-functions, reductions and hodograph solutions of the $r$ th dispersionless modified KP and Dym hierarchies, J. Phys. A: Math. Gen. 37 (2004), 11191-11221, nlin.SI/0405028.

[27] Mañas M., Martínez Alonso L., Medina E., Reductions and hodograph solutions of the dispersionless KP hierarchy, J. Phys. A: Math. Gen. 35 (2002), 401-417.

[28] Pavlov M.V., Algebro-geometric approach in the theory of integrable hydrodynamic type systems, Comm. Math. Phys. 272 (2007), 469-505, nlin.SI/0603054.

[29] Riley A., Strachan I.A.B., A note on the relationship between rational and trigonometric solutions of the WDVV equations, J. Nonlinear Math. Phys. 14 (2007), 82-94, nlin.SI/0605005.

[30] Takasaki K., Generalized string equations for double Hurwitz numbers, J. Geom. Phys. 62 (2012), 11351156, arXiv:1012.5554.

[31] Takasaki K., Takebe T., Integrable hierarchies and dispersionless limit, Rev. Math. Phys. 7 (1995), 743-808, hep-th/9405096.

[32] Takasaki K., Takebe T., Löwner equations, Hirota equations and reductions of the universal Whitham hierarchy, J. Phys. A: Math. Theor. 41 (2008), 475206, 27 pages, arXiv:0808.1444.

[33] Takasaki K., Takebe T., Radial Löwner equation and dispersionless mcKP hierarchy, nlin.SI/0601063.

[34] Takebe T., Teo L.-P., Zabrodin A., Löwner equations and dispersionless hierarchies, J. Phys. A: Math. Gen. 39 (2006), 11479-11501, math.CV/0605161.

[35] Tsarev S.P., Classical differential geometry and integrability of systems of hydrodynamic type, in Applications of Analytic and Geometric Methods to Nonlinear Differential Equations (Exeter, 1992), NATO Adv. Sci. Inst. Ser. C Math. Phys. Sci., Vol. 413, Kluwer Acad. Publ., Dordrecht, 1993, 241-249, hep-th/9303092.

[36] Tsarev S.P., On Poisson bracket and one-dimensional systems of hydrodynamic type, Soviet Math. Dokl. 31 (1985), 488-491.

[37] Yu L., Waterbag reductions of the dispersionless discrete KP hierarchy, J. Phys. A: Math. Gen. 33 (2000), $8127-8138$. 